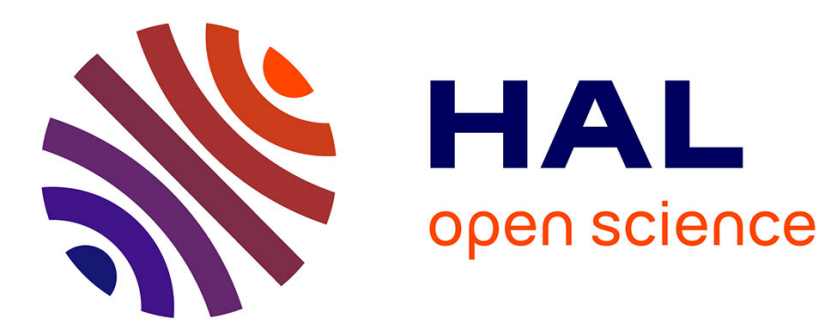

\title{
Terrain obscuration managment for multiple ground target tracking
}

Benjamin Pannetier, Michèle Rombaut

\section{To cite this version:}

Benjamin Pannetier, Michèle Rombaut. Terrain obscuration managment for multiple ground target tracking. FUSION 2007 - 10th International Conference on Information Fusion, Jul 2007, Québec, QC, Canada. s.p. hal-00153313

\section{HAL Id: hal-00153313 \\ https://hal.science/hal-00153313}

Submitted on 8 Dec 2007

HAL is a multi-disciplinary open access archive for the deposit and dissemination of scientific research documents, whether they are published or not. The documents may come from teaching and research institutions in France or abroad, or from public or private research centers.
L'archive ouverte pluridisciplinaire HAL, est destinée au dépôt et à la diffusion de documents scientifiques de niveau recherche, publiés ou non, émanant des établissements d'enseignement et de recherche français ou étrangers, des laboratoires publics ou privés. 


\section{Terrain obscuration managment for multiple ground target tracking}

\author{
Benjamin Pannetier \\ Information Processing and Modeling Department \\ ONERA \\ 29 avenue de la Div. Leclerc, 92322 Châtillon, France \\ Email: benjamin.pannetier@onera.fr
}

\author{
Michele Rombaut \\ GIPSA \\ Université de Grenoble \\ 46 avenue Felix Viallet, 38031 Grenoble, France \\ Email: michele.rombautelis.inpg.fr
}

\begin{abstract}
Multiple ground targets tracking with a GMTI (Ground Moving Target Indicator) sensor is considered a challenging problem in order to establish battlefield assessment. An IMM algorithm with a variable structure is adapted to the road network and used to track multiple manoeuvring ground targets. However, the case of undetected targets due to terrain elevation or Doppler obscuration was not taken into account in our tracking process. In this paper, we present our approach to track ground targets with the possibility for the target to be undetected. The perceivability probability is computed to update the estimated state and a "sentinel" concept is used to palliate the association ambiguities when several targets enter and exit the same terrain mask.
\end{abstract}

Keywords: tracking, GMTI, multiple targets, VS IMM under constraint, negative information.

\section{INTRODUCTION}

Tracking ground targets with a GMTI (Ground Moving Target Indicator) sensor is a particular problem because of the high traffic density and the large number of false alarms, that bring about a significant quantity of data. If we add strong and fast target manoeuvres, target tracking is compromised due to the association problem and manoeuvre problems. In a GMTI surveillance context, we have proposed to fuse the road network information with the MTI reports in order to improve the track quality. Based on the road segments positions, dynamic models under road constraint are built and an optimized projection technique of the estimated states is proposed to keep each target position and heading on the road. A VS-IMM (Variable Structure Interacting Multiple Model) filter is built using this projection approach [1]. The set of models used in the filter is adjusted sequentially according to the target positions and to the road network configuration. In a multiple target scenario, we have adapted the SB-MHT (Structured Branching - Multiple Hypotheses Tracking) to take into account the target manoeuvres and the detection probability of each ground target. This algorithm called VS IMMC SB-MHT was efficient if only the targets were always detectable by the sensor. An illustration has been given with 20 targets which manoeuvre on the road network and/or leave the road network [2]. However, the detection probability varies with the network configuration, the terrain elevation and the halt of the target. The performances of the algorithm have been degraded when, for example, the targets were masked by the terrain and moved in the mask responsible for the loss of the tracks.

According to the geographic information and the sensor location, it is possible to know the target capacity to be detected by the sensor. So, this paper focuses on the multiple targets tracking which take into account both positive information (MTI reports are present in the validation gate) as well as negative information (no MTI report is validated). The negative information is the undetection, which results, for instance, of the ground target deliberately stopping.

In the literature, Kirubarajan et al. consider this information in [3], and propose to palliate the stop manoeuvre by introducing a stop motion model in a usual IMM. This technique was extended and introduced in the VS IMMC SB-MHT by taking into account the road network in the ground target tracking process. In addition, due to the terrain elevation and the road network configuration, the ground targets can be undetected and moving on the road network. In fact, the terrain elevation or vegetation generate terrain masks by which the targets are hidden. Connare and Blasch in [4] deal with the undetection in a special case where a tank formation is under trees. The authors propose to measure the distance and bearing information of all the targets within the group as they are related to each other. Then, when one of the tanks is obscured by the vegetation, the tracking algorithm will recall from past measurements the distance and bearing information of the missing target and assume that the same positional relationships are valid at the current time. Despite the track maintenance of the undetected targets this approach cannot be used for only one target or several targets who move on the same road. Finally, the GMTI sensor does not detect the ground targets when their radial velocity falls under the Minimum Detectable Velocity (MDV) fixed by the sensor. Nevertheless, in their recent article [5], Lin et al. propose to combine the sensor detection probability $P_{D}$ with the probability that the target radial velocity is inferior to the MDV. The radial velocity is estimated according to the target estimated velocity heading and the sensor location.

Consequently, if the terrain obscuration condition is not taken into account, the VS IMMC loses the tracks when the targets are masked or undetected due to their radial velocity, because the stop motion model of the VS IMMC is activated 
despite the target movement. We propose to introduce in the VS IMMC the event on the perceivability target. Dezert et al. proposed this idea in [6]. However, the perceivability probability does not take into account the sensor MDV and the terrain obscuration. Therefore, we introduced in this paper the prior information on the target perceivability to keep the tracks and not activate the stop motion model when the targets are masked. That implies the track maintenance when the targets are masked. However if several targets are in the same terrain mask and leave it through several exits, the used SB-MHT take an arbitrary decision to associate a track to a MTI report. In fact we do not have, in our sensor, the HRRR (High Range Resolution Radar) used in [7] in order to distinguish the targets from each other. Thus, the same track represents different target trajectories and deteriorates the situation assessment. That is why we propose to use a concept of "sentinel" which is applied to evaluate the track association ambiguity at the exits of the mask.

This paper is organized as follows : in section II, we give a brief description of the measurement model and of the target constrained motion model. In section III, we present the perceivability probability and its introduction in the target tracking process. The sentinel concept is presented in section IV. Finally, in section V, we illustrate our approach on a complex scenario with 6 targets.

\section{TARGET MOTION AND MEASUREMENT MODEL}

\section{A. Introduction}

Usual target tracking algorithms are based on the Kalman filter. Since several years, in the ground target tracking domain, the kalman filter is evolved by considering the contextual information in the tracking process. For instance, Kirubarajan et al. proposed to use in [8] the road segment location in order to modelized the dynamic of a target on the road. The road network is considered here as a priori information to be integrated in the tracker system. The map information is contained in a GIS (Geographic Information System). It contains the information about the road network location and the DTED (Digital Terrain Elevation Data). In the following, the stochastic target constrained and the Kalman filter under constraints are described in addition to the GIS description.

\section{B. GIS description}

The GIS used in this work contains the following information: the segmented road network and DTED. Each road segment is expressed in the WGS84 system. The road network is connex and each road segment is indexed by the road section they belong to. A road section is defined by a connected road segments set delimited by a road end or a junction. Starting from the DTED a Delaunay triangulation is applied. Then, knowing the sensor location a Z_Buffer technique is computed to define the triangles seen or not seen by the sensor. Consequently, at the current time it is possible to obtain a picture $I$ which describes the pixels seen or not by the sensor. You can see on the figure 1, the terrain elevation data in blue

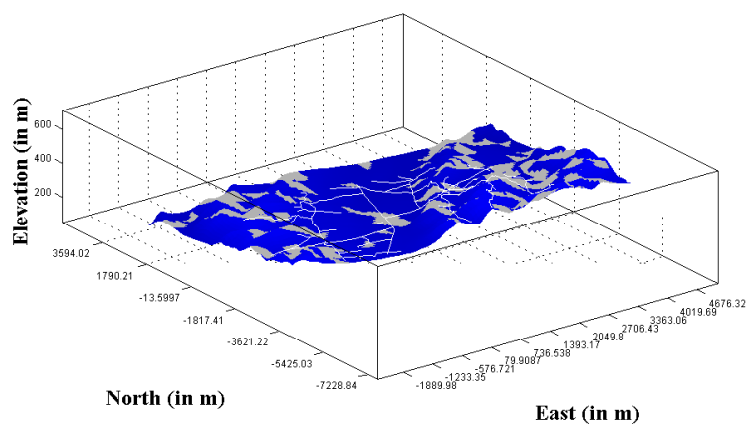

Fig. 1. Terrain masks visualisation

with the triangles in grey who represent the terrain masks (i.e. the areas without any detection).

At the beginning of a surveillance battlefield operation, a Topographic Coordinate Frame (noted TCF) and its origin $O$ are chosen in the manner that the axes $X, Y$ and $Z$ are respectively oriented in the east, north and up local direction. Now, the target tracking process is carried out in the TCF.

\section{Target state under constraint}

The target state at the current time $k$ is defined in the local coordinate frame by :

$$
x(k)=\left[\begin{array}{llll}
\mathrm{x}(k) & \dot{\mathrm{x}}(k) & \mathrm{y}(k) & \dot{\mathrm{y}}(k)
\end{array}\right]^{\prime}
$$

where the couples $(\mathrm{x}(k), \mathrm{y}(k))$ and $(\dot{\mathrm{x}}(k), \dot{\mathrm{y}}(k))$ define respectively the target location and velocity in the cartesian coordinates frame. The altitude component is not considered because the effect of this one is supposed negligible on the estimate state. The dynamics of the target evolving on the road network are modelized by a first order system. The target state under the road segment $s$ is defined by $x_{s}(k)$ where the target position $\left(\mathrm{x}_{s}(k), \mathrm{y}_{s}(k)\right)$ belongs to the road segment and the corresponding velocity vector $\left(\dot{x}_{\mathcal{S}}(k), \dot{\mathrm{y}}_{\mathcal{S}}(k)\right)$ is in the road segment $s$ direction. Therefore, the target constraint state $x_{s}(k)$ is defined by the following constraint:

$$
\left\{\begin{array}{l}
a \cdot \mathrm{x}_{s}(k)+b \cdot \mathrm{y}_{s}(k)+c=0 \\
\left\langle\left[\begin{array}{ll}
\dot{\mathrm{x}}(k) & \dot{\mathrm{y}}(k)
\end{array}\right]^{\prime} \mid \vec{n}_{s}\right\rangle=0
\end{array}\right.
$$

where the indexes $a, b$ and $c$ are the coefficients of the line associate to the road segment $s$ and $\vec{n}_{s}$ is the normal vector to the road segment $s$. The constraint can be expressed as follows:

$$
\tilde{D} \cdot x_{s}(k)=L
$$

with $\tilde{D}=\left[\begin{array}{cccc}a & 0 & b & 0 \\ 0 & a & 0 & b\end{array}\right]$ and $L=\left[\begin{array}{c}-c \\ 0\end{array}\right]$.

The event that the state belongs to the road segment $s$ is noted $e_{s}(k)=\{x(k) \in s\}$. Knowing the event $e_{s}(k)$ and according to a motion model $\mathrm{M}_{i}$ the dynamics of the target can be improved by considering the road segment $s$. Due to the precision of the GMTI sensor and the long time scan period, the chosen motion models are basics. It is about $r$ constant 
velocity motion models different on the standard deviation values. However the proposed approach is valid for much more complicated motion models like constant acceleration or coordinated turn motion models. It follows that :

$$
x_{s}(k)=F_{s, i}\left(\Delta_{k}\right) \cdot x_{s}(k-1)+\Gamma\left(\Delta_{k}\right) \cdot \nu_{s, i}(k)
$$

where $\Delta_{k}$ is the time of sampling, the matrix $F_{s, i}$ is the state transition matrix associated to the road segment $s$ and described in [11] and is adapted to a motion model $\mathrm{M}_{i}$, the variable $\nu_{s}(k)$ is a white noise gaussian process and its associated covariance $Q_{s, i}(k)$ is built in the manner that the standard deviation along the road segment is higher than the standard deviation in the orthogonal direction. Consequently the covariance matrix $Q_{s, i}$ is defined by :

$$
Q_{s, i}(k)=R_{\vartheta_{s}} \cdot\left[\begin{array}{cc}
\sigma_{d}^{2} & 0 \\
0 & \sigma_{n}^{2}
\end{array}\right] \cdot R_{\vartheta_{s}}^{T}
$$

where the matrix $R_{\vartheta_{s}}$ is the rotation matrix associate to the $s$ road segment direction $\vartheta_{s}$ defined in the plane $(O, X, Y)$. The matrix $\Gamma\left(\delta_{k}\right)$ is defined in [12]. We call $Z(k)$ the set of measurements obtained at the current time.

We define $M_{s}^{i}(k)=\left\{M^{i}(k) \cap \epsilon_{s}(k)\right\}$ the event that the target is following a dynamic according to the motion model $M^{i}$ and moves on the road segment $s$. So, the state probability density function (i.e. pdf) considering the measurements set $Z^{k}$ and the event $M_{s}^{i}(k)$ is denoted :

$$
p\left\{x(k) \mid Z^{k}, M_{s}^{i}(k)\right\}
$$

The state $x_{i}(k)$ is a Gaussian random variable defined by its estimated mean $\hat{x}_{i}(k \mid k)$ and its estimated covariance $\mathrm{P}_{i}(k \mid k)$ (both obtained using a model based filter). Under the road constraint, the estimated state $\hat{x}_{s, i}(k \mid k)$ is therefore the state obtained by the maximization of pdf (6) given the event $M_{s}^{i}$. Finally, under the gaussian assumption of the Kalman filter the analytic expression of the constraint estimate state associate to the motion model $\mathrm{M}^{i}$ is obtained by calculating the Lagrangian of (6) under the constraint (3). The expressions of constraint estimated state and it covariance are given in [13].

We have presented the taget motion model and the estimated state on a road segment $s$ according to the dynamic $\mathbf{M}^{i}$. However the road network is composed of several road segments and a ground target has several motion models. In the paper [2] we have presented our approach for tracking several targets which are manoeuvring on the road network. So we have considered an IMM (Interacting Multiple Model) with a variable structure to adapt the constraint motion models set to the road network configuration. We have extended this approach with the targets of interest who can leave the road network. The VS IMMC combined with a SB-MHT has been used on real data. The obtained results are better than the usual approach without constraint and/or without several motion models under constraint, in order to palliate the targets manoeuvres on the road. However, the weakness of our algorithm is, it does not take in account the non-detection of the targets expected by a random event characterized by the sensor detection probability $P_{D}$. In the section III, we enlarge the algorithm by tacking in account the non-detections due to the stop of the targets, the range radial velocity less than the MDV and the terrain masks.

\section{Measurement model}

According to the NATO GMTI formats, the MTI reports are expressed in WGS84 coordinates system. All MTI reports are export for each tracking station in the TCF. A MTI measurement $z$ at the current time $k$ is given in the TCF by:

$$
z(k)=\left[\begin{array}{lll}
\mathrm{x}(k) & \mathrm{y}(k) & \dot{\rho}_{m}(k)
\end{array}\right]^{\prime}
$$

The couple $(\mathrm{x}(k), \mathrm{y}(k))$ is the MTI coordinate in the local frame $(0, X, Y)$ and $\dot{\rho}_{m}$ is the associated modified range-rate measurement expressed in the TCF:

$$
\dot{\rho}_{m}(k)=\frac{\mathrm{x}(k) \cdot \dot{\mathrm{x}}(k)+\mathrm{y}(k) \cdot \dot{\mathrm{y}}(k)}{\sqrt{\mathrm{x}^{2}(k)+\mathrm{y}^{2}(k)}}
$$

We don't consider the range-rate $\dot{\rho}$ obtain directly in the sensor frame because the range-rate is correlated to the MTI location components. In the literature it exists several techniques to uncorrelate the range-rate from the location components like the SEKF from Wang et al. in [9] which use a Cholesky decomposition in order to do it. Nevertheless, we prefer to use the AEKF (Alternative Extended Kalman Filter) presented by Bizup and Brown described in the paper [10]. This last one is very simple to compute because the authors propose only to use an alternative linearization of the EKF (Extended Kalman Filter). For our problem, the alternative linearization of the observation function is verified only if we consider the range-rate in the local frame TCF. Then, the measurement equation is given according to the AEKF, by:

$$
z(k)=H(k) \cdot x(k)+\nu(k)
$$

with $\nu(k)$ a zero-mean white gaussian noise vector and $H(k)$ defined by:

$$
H(k)=\left[\begin{array}{cccc}
1 & 0 & 0 & 0 \\
0 & 0 & 1 & 0 \\
0 & \frac{\partial \dot{\rho}_{m}(k)}{\partial \mathrm{x}} & 0 & \frac{\partial \dot{\rho}_{m}(k)}{\partial \mathrm{y}}
\end{array}\right]
$$

The explicit expression of (10) is given in [10]. In the following of the article, the event $\theta^{k, l}$ is associated to the $l^{t h}$ sequential measurements $Z^{k, l}$ and represents the set of measurement generated by the target (it exists a subsequence $n$ and a measurement $i$ in the manner that $\left.Z^{k, l}=\left\{Z^{k-1, n}, \ldots, z_{i}(k)\right\}\right)$.

\section{PERCEIVABILITy PROBABILITy}

In this part, based on the work of Dezert et al., we introduce the event that the targets of a track $\theta^{k, l}$ is perceivable or not by the GMTI sensors. 


\section{A. Perceivability probability in the target tracking process}

At time $k$, the target state probability is represented by the following exhaustive and exclusive events :

$$
\begin{gathered}
O_{k}=\{\text { target is perceivable }\} \\
\bar{O}_{k}=\{\text { target is unperceivable }\}
\end{gathered}
$$

here, $O_{k}$ will denote both the target can be detected by the sensor and the random event. By introduce the both event in the conventional IMM, we obtain a new formulation of the likelihood function. But the perceivability event does not take in account the non-detection due to the target stop. That is why, we used the IMM proposed by Kirubarajan et al. in [3], where the authors introduce a stop motion model noted $\mathrm{M}_{\mathrm{s}}^{\mathrm{o}}$. In the VS IMMC, we have for each motion model beyond $r+1$ motions models $(\forall i \in\{0, \ldots, r\})$ :

$$
\Lambda_{i}(k)=p\left\{z(k) \mid Z^{k-1, n}, \theta^{k, l}, M_{s}^{i}(k)\right\}
$$

where $z(k)$ is the MTI report define in (7), $Z^{k-1, n}$ is a particular sequence of previous measurement. Now, according to the total probability rules, we introduce the event that the target is detected (i.e. $\{m=1\}$ ) or not (i.e. $\{m=0\}$ ) and the events $O_{k}$ and $\bar{O}_{k}$. We obtain from (12):

$$
\begin{aligned}
\Lambda_{i}(k) \quad & =p\left\{z(k), m=1, O_{k} \mid Z^{k-1, n}, \theta^{k, l}, \mathrm{M}_{s}^{i}(k)\right\} \\
& +p\left\{z(k), m=1, \bar{O}_{k} \mid Z^{k-1, n}, \theta^{k, l}, \mathrm{M}_{\mathcal{s}}^{i}(k)\right\} \\
& +p\left\{z(k), m=0, O_{k} \mid Z^{k-1, n}, \theta^{k, l}, \mathrm{M}_{\mathcal{s}}^{i}(k)\right\} \\
& +p\left\{z(k), m=0, \bar{O}_{k} \mid Z^{k-1, n}, \theta^{k, l}, \mathrm{M}_{\mathcal{s}}^{i}(k)\right\}
\end{aligned}
$$

However, an unperceivable target can not be detected. so the event $\left\{m=1, \bar{O}_{k}\right\}$ is equal to

oslash. According to the Kirubarajan approach, we distinguish the motion model STOP noted $\mathrm{M}^{\mathrm{O}}$ from the set of motion models. The event $\left\{M_{s}^{0}, m=1\right\}$ is equal to $\oslash$, because the STOP motion model must not be activated if there is at least one detection. By using the Bayes rule, we find the new expression of the likelihood function $(\forall i \in\{0, \ldots, r\})$ :

$$
\begin{aligned}
\Lambda_{i}(k) & =\left(1-\delta_{m, 0}\right) \cdot P_{D} \\
& \cdot p\left\{z(k) \mid Z^{k-1, n}, \theta^{k, l}, M_{s}^{i}(k), O_{k}\right\} \\
& \cdot P\left\{O_{k} \mid Z^{k-1, n}, \theta^{k, l}, M_{s}^{i}(k)\right\} \\
& +\left(1-P_{D}\right) \cdot \delta_{m, 0} \cdot P\left\{O_{k} \mid Z^{k-1, n}, \theta^{k, l}, M_{s}^{i}(k)\right\} \\
& +\delta_{m, 0} \cdot\left(1-P\left\{O_{k} \mid Z^{k-1, n}, \theta^{k, l}, M_{s}^{i}(k)\right\}\right)
\end{aligned}
$$

and

$$
\Lambda_{0}(k)=\delta_{m, 0} \cdot P\left\{O_{k} \mid Z^{k-1, n}, \theta^{k, l}, M_{s}^{0}(k)\right\}
$$

where $\delta_{m, 0}$ is the Kronecker function equal to unity if there is no detection $(m=0)$. The probability to obtain at least one measurement is equal to the detection probability (i.e. $P\left\{m=1 \mid Z^{k-1, n}, \theta^{k, l}, M_{s}^{i}(k), O_{k}\right\}=$ $\left.\left(1-\delta_{m, 0}\right) \cdot P_{D}\right)$ in opposition to obtain no measurement (i.e. $P\left\{m=0 \mid Z^{k-1, n}, \theta^{k, l}, M_{\mathcal{s}}^{i}(k), O_{k}\right\}=\left(1-\delta_{m, 0}\right)$. $\left.\left(1-P_{D}\right)\right)$.

\section{B. Unperceivability probability calculation}

In this part, we present the different reasons due to the target undetection. In order to know the perceivability probability we calculate the unperceivability probability. We consider in this paper that the target is unperceivable by the GMTI sensor because the target is either hidden by the terrain elevation (this masks is noted $\mathrm{Ma}_{1}$ ), or either the radial target velocity is less than the MDV (this masks is noted $\mathrm{Ma}_{2}$ ). So for each motion model we calculate the unperceivability probability $(\forall i \in\{0, \ldots, r\})$ :

$$
\begin{aligned}
& P\left\{\bar{O}_{k} \mid Z^{k-1, n}, \theta^{k, l}, M_{s}^{i}(k)\right\}= \\
& P\left\{M_{a_{1}}(k) \cup M_{a_{2}}(k) \mid Z^{k-1, n}, \theta^{k, l}, M_{s}^{i}(k)\right\}
\end{aligned}
$$

where $M a_{1}$ and $M a_{2}$ are the events associate the unperceivability due to the mask $\mathrm{Ma}_{1}$ and $\mathrm{Ma}_{2}$ respectively. The problem is the both events are not independents because the mask $\mathrm{Ma}_{1}$ depends on the sensor location to compute the binary picture $I$ of terrain masks and the mask $\mathrm{Ma}_{2}$ depends on the sensor line of sight, then the sensor location. However, our goal is only to know the target perceivability (the target is undetected either by a mask $\mathrm{Ma}_{1}$, either by a mask $\mathrm{Ma}_{2}$ or the both), that is why we use the max operator to compute the probability (16). It follows :

$$
\begin{aligned}
& P\left\{\bar{O}_{k} \mid Z^{k-1, n}, \theta^{k, l}, M_{s}^{i}(k)\right\}= \\
& \max \left(P\left\{M_{a_{1}}(k) \mid Z^{k-1, n}, \theta^{k, l}, M_{s}^{i}(k)\right\}, \cdots\right. \\
& \left.P\left\{M_{a_{2}}(k) \mid Z^{k-1, n}, \theta^{k, l}, M_{s}^{i}(k)\right\}\right)
\end{aligned}
$$

The prior unperceivability probability of the mask due to the terrain elevation is evaluated thanks to the digital terrain elevation data called DTED. Knowing the location of the sensor and the DTED, it is possible to compute a binary picture $I$, which indicate the non-detection areas. We use a Z-buffer algorithm in order to know if a pixel from $I$ is seen or not by the sensor. Starting with the predicted state $\hat{x}_{i, s}(k \mid k-1)$ under constraint from each motion model and its associate covariance $\mathrm{P}_{i, s}(k \mid k-1)$, we are able to obtain the target location $\left(c_{\mathrm{x}}, c_{\mathrm{y}}\right)$ and the location covariance $\mathrm{P}_{\text {loc }}$ in the picture $I$, according to the transformation function $T_{T C F \rightarrow I}$ between the real location in the $T C F$ and the picture I. Due to the uncertainty on the target location, we propose to take in account a neighbourhood of $\left(c_{\mathrm{x}}, c_{\mathrm{y}}\right)$ in order to know if the target is unperceivable. Finally, the prior unperceivability probability $\mathrm{M}_{\mathrm{a}_{1}}$ is equal to:

$$
\begin{aligned}
& P\left\{M_{a_{1}}(k) \mid Z^{k-1}, \theta^{k, l}, M_{s}^{i}(k)\right\} \\
& =\sum_{i=1}^{L} \sum_{j=1}^{L} H_{\hat{x}_{i, s}(k \mid k-1)}(i, j) \times \cdots \\
& I\left(c_{x}+i-\left\lfloor\frac{L}{2}\right\rfloor-1, c_{y}+j-\left\lfloor\frac{L}{2}\right\rfloor-1\right)
\end{aligned}
$$

where $H_{\hat{x}_{i, s}(k \mid k-1)}(i, j)$ is the pdf value of the Gaussian distribution at the pixel $(i, j)$ : 


$$
\begin{aligned}
& H_{\hat{x}_{i, s}(k \mid k-1)}(i, j)=\frac{1}{\sqrt{|2 \cdot \pi \cdot \operatorname{Pp}|}} \times \exp (\cdots \\
& \left.-\frac{1}{2} \cdot\left(\left[\begin{array}{l}
i \\
j
\end{array}\right]-\left[\begin{array}{l}
c_{x} \\
c_{y}
\end{array}\right]\right)^{\prime} \cdot \mathrm{Pp}^{-1} \cdot\left(\left[\begin{array}{l}
i \\
j
\end{array}\right]-\left[\begin{array}{l}
c_{x} \\
c_{y}
\end{array}\right]\right)\right)
\end{aligned}
$$

The value $L$ is the gate equal to the maximum standard deviation of $\mathrm{P}_{l o c}$ and $\lfloor\cdot\rfloor$ symbolizes the integral part.

We calculate now the prior unperceivability probability that the target can not be detected due to its radial velocity inferior to the MDV. According to a Gaussian distribution, the predicted radial velocity, noted $\dot{\rho}_{i}(k \mid k-1)$, from each track and each motion model given in [5] is evaluated and the probability that the radial velocity is under the MDV can be calculated.

$$
\begin{aligned}
& P\left\{M_{a_{2}}(k) \mid Z^{k-1, n}, \theta^{k, l}, M_{s}^{i}(k)\right\}= \\
& P\left\{0<\dot{\rho}_{i}(k \mid k-1)<M D V \mid Z^{k-1, n}, \theta^{k, l}, M_{s}^{i}(k)\right\}
\end{aligned}
$$

\section{Principle}

$\begin{array}{ccc}\text { If the perceivability } & \text { probability } \\ P\left\{O_{k} \mid Z^{k-1, n}, \theta^{k, l}, M_{s}^{i}(k)\right\}(\forall i \in\{0, \ldots, r\}) & \text { is equal }\end{array}$ to one, we find the likelihood function given in [3], i.e. the target is perceivable and if there is no detection, the stop motion model is activated. On the other hand, if this perceivability probability for each motion model is equal to zero, the stop likelihood function (15) is equal to zero (that brings about the non activation of the stop motion model) and the others motion model likelihood functions (14) are equi-probable that brings about the keep of track during its masking. Now, we must calculate the perceivability probability from each motion model.

Consequently the prior perceivability probability (16) can be calculated for each motion model. In the particular case, where the system track only one target in a terrain mask, the proposal approach is sufficient for the track maintenance. In the terrain mask, the perceivability probability (16) is equal to zero and the predicted states are propagated and each road section during the masking.

\section{SENTINEL SOLUTION FOR MULTIPLE TARGET TRACKING}

However, when several targets are in the same mask, the kinematics information is not sufficient to discriminate the targets in the mask. In fact, if several predicted states associated to different tracks are located on the same exit of the mask, the SB-MHT will take an arbitrary decision (the most probable association) to attach the new MTI report to only one tack. When two tracks, noted $T^{k, 1}$ and $T^{k, 2}$, exit from a same terrain mask their perceivability probability from each motion model tends to one. That brings about the stop motion model activation for the both tracks. If a MTI reports appears, the VS IMMC SB-MHT extends only one track, for instance $T^{k, 1}$. However the targets associate to the tracks $T^{k, 1}$ and $T^{k, 2}$ can manoeuvre in the terrain mask and there is no guarantee that the new MTI report is originated from the target associate the track $T^{k, 1}$. In order to modelize the association ambiguity, we propose to build a new track $T^{k, 3}$, starting from the new MTI report, in which it is possible to find the information that this new track $T^{k, 3}$ is originated either from $T^{k, 1}$ or $T^{k, 2}$. For this, it is necessary to place the tracks $T^{k, 1}$ and $T^{k, 2}$ in a "sentinel" configuration. Then, like a sentinel, the tracks $T^{k, 1}$ and $T^{k, 2}$ are fixed (there is no maintenance) and waiting for all MTI reports in their neighbourhood.

\section{A. Sentinel Activation}

To declare a track as "sentinel", we compute the combined perceivability probability of a target as following:

$$
\begin{aligned}
& P\left\{O(k) \mid Z^{k-1, n}, \theta^{k, l}\right\}= \\
& \sum_{i=0}^{r} P\left\{O(k) \mid Z^{k-1, n}, \theta^{k, l} ; M_{s}^{i}\right\} \cdot \mu_{i}(k \mid k-1)
\end{aligned}
$$

where the perceivability probability $P\left\{O(k) \mid Z^{k-1, n}, \theta^{k, l} ; M_{s}^{i}\right\} \quad$ is complementary the unperceibaility probability (16) for each motion model and $\mu_{i}(k \mid k-1)$ is the predicted mode probability given in [14].

Step 1: Sentinel configuration.

If the probability (21) is less than a given threshold $\gamma$, the associated track is placed in a inactive sentinel configuration. Note that the track is always in the terrain mask and the predicted states are always propagated. Then, when the track in the inactive sentinel configuration leaves the terrain mask, the combined perceivability probability increases. In the figure IV-A, we can see a track approaching a terrain mask. At time $k$, the combined perceivability probability function is high and the predicted state is updated. But at time $k+1$ the combined perceivability probability low and the track is placed in a sentinel configuration.

Step 2: Sentinel activation.

If the probability (21) is higher than the threshold $\gamma$ the sentinel becomes active. This is the case at each mask exit. This special state, called sentinel, is waiting for the MTI report, like a cat who is waiting for a mouse to come out of cover. In the figure IV-A, according to the road network configuration the track in sentinel configuration is duplicated in the intersection at time $k+2$. the right track leave the terrain mask and the combined perceivability increases and exceeds the threshold $\gamma$. Then the sentinel becomes active, whereas the down track is always in the terrain mask, its combined perceivability is always inferior than the given threshold $\gamma$ and the track stays in a inactive sentinel configuration.

Step 3: Sentinel association.

If the activated sentinel validates a new MTI report, a new track is build starting from this MTI report with the information that this new track comes from the track associated to the sentinel.

\section{B. Decision}

Finally, it is possible to associate in a new track $T^{k, N}$ the information that it is originated from the track $T^{k, l}$ according to the sentinel validation. For each hypothesis, we evaluate the 


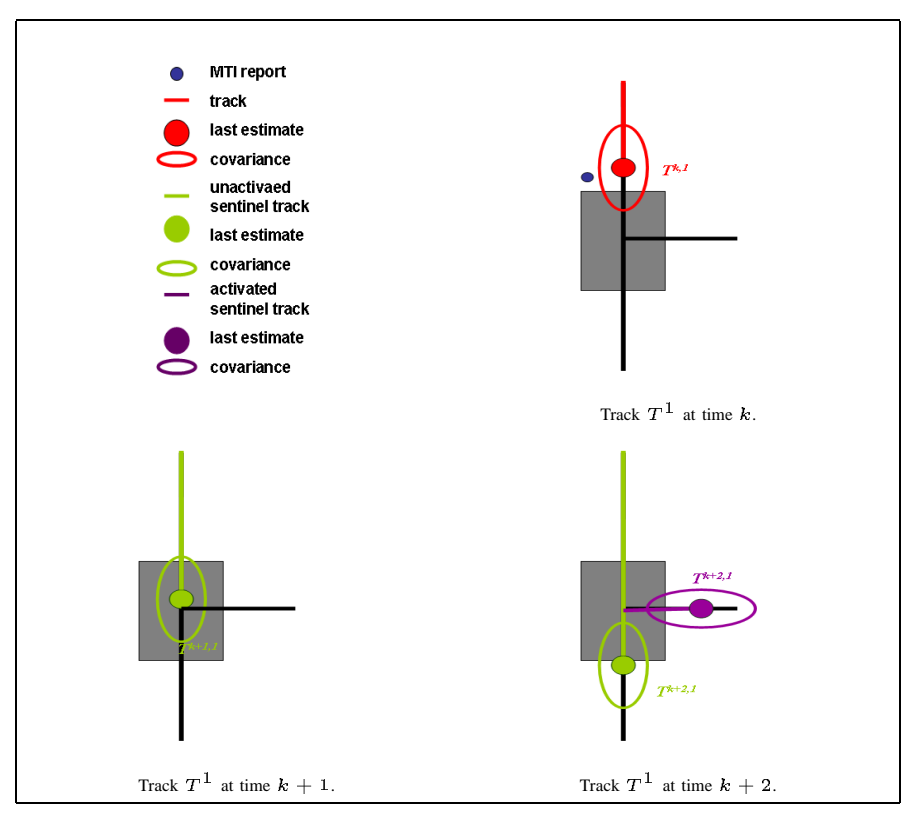

Fig. 2. Example of sentinel activation

probability that the new track comes from the sentinel which can be written:

$$
\begin{aligned}
& P\left\{T^{k, N} \rightarrow T^{k, l} \mid Z^{k}\right\}= \\
& P\left\{x(k) \mid Z^{k}, \theta^{k, l}\right\} \cdot P\left\{\operatorname{Sent}\left(T^{k, l}\right) \geq t_{k} \mid Z^{k}\right\}
\end{aligned}
$$

the first term of the expression (22) is the global probability track given directly by the SB-MHT. The second term represents the probability that the sentinel is still activated at the current time $t_{k}$. This probability has not a physical sense. Then we can said this probability decreases linearly. In our case we compare the activated sentinel to a radioactive element who marks the new tracks with the associated old tracks. Then the probability that the sentinel is always in life at the current time is:

$$
P\left\{\operatorname{Sent}\left(T^{k, l}\right) \geq t_{k} \mid Z^{k}\right\}=\exp \left(-\frac{t_{k}-t_{0}}{\Delta \text { Sent }}\right)
$$

where $t_{0}$ is the first activation time and $\Delta$ Sent is the average sentinel activation time. If in a new track $T^{k, N}$ the probability (23) of an old track $T^{k, l}$ is important, we decide that the track $T^{k+1, N}$ is the track continuity of the track $T^{k, l}$. Otherwise no decision is taken because of several old tracks continuation ambiguities.

\section{Modification of the motion model in a terrain mask}

If the tracks are masked by a short terrain mask, the combined perceivability probability (21) is higher than the given threshold $\gamma$ and the tracks does not become a sentinel. On the contrary, for the large terrain masks, the combined perceivability probability becomes less than $\gamma$ and the tracks become inactivated sentinels. If there is no modification of the motion models, the covariance matrix grow up until the sentinel is activated. Consequently, the activated sentinel
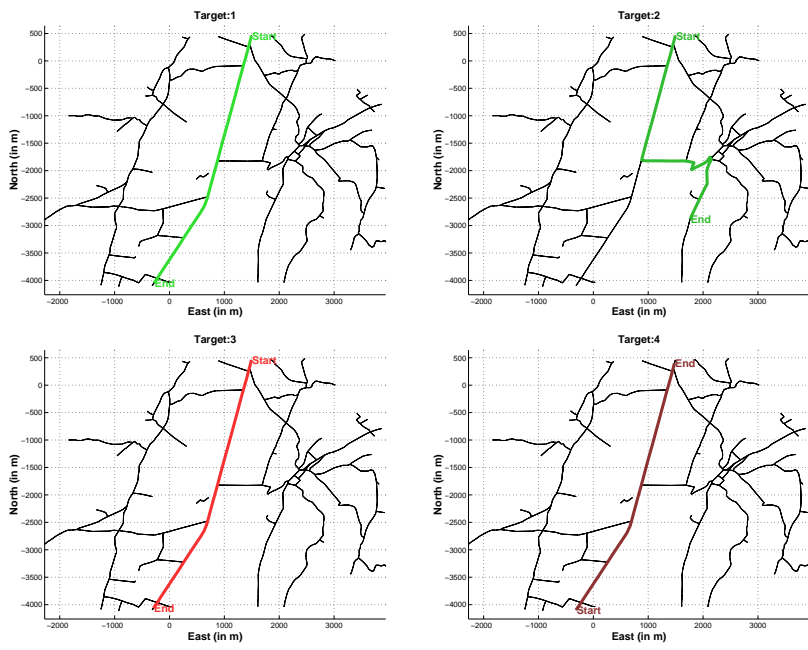

Targets
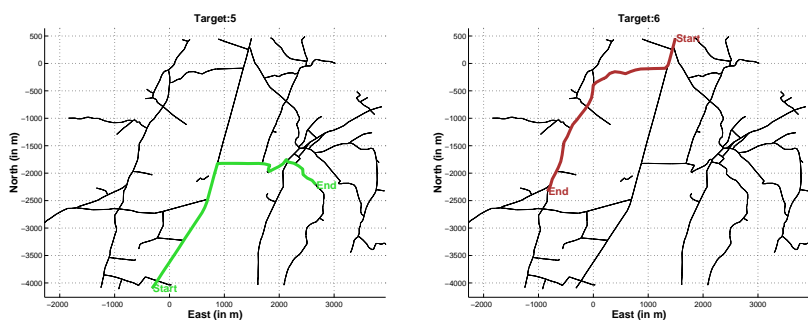

Fig. 3. The sixth ground targets trajectories

can validated far MTI reports because of the big estimated covariance. That is why the motion model (4) is replaced by $(\forall i \in\{0, \ldots, r\})$ :

$$
x_{s}(k)=F_{s, i}\left(\Delta_{k}\right) \cdot x_{s}(k-1)+\delta_{\text {Sent }, 0} \cdot \Gamma\left(\Delta_{k}\right) \cdot \nu_{s, i}(k)
$$

where $\delta_{\text {Sent }, 0}$ is the Kronecker symbol equal to one or zero if the track becomes respectively an active/inactive sentinel or not.

\section{Simulation And RESUlts}

\section{A. Scenario description}

We consider 6 targets moving in the Topographic Coordinate Frame. The targets 1, 3 and 4 move on the same road with a constant velocity (3). The targets 2 and 5 move on the road network in order to take another road in an intersection. The targets 1,2 and 4 start in a close formation and cross the targets 2 and 5 . The target 6 does not cross the others target but decelerates in order to stop during 30 seconds.

\section{B. Sensor description}

The 6 targets are tracked by a GMTI sensor at $0.1 \mathrm{~Hz}$ with $20 \mathrm{~m}, 0.0008 \mathrm{rad}$ and $1 \mathrm{~m} / \mathrm{s}$ range, cross-range and range-rate measurement standard deviation respectively. The detection probability $P_{D}$ is equal to 0.9 and the MDV fixed at $1 \mathrm{~m} / \mathrm{s}$. The sensor is located at $60 \mathrm{~km}$ from the $T C F$ origine at 4000 $m$ in elevation and moves in the west direction. The terrain masks are computed at each time according the Digital Terrain Elevation Data (the yellow triangles in the figure 4). In addition 


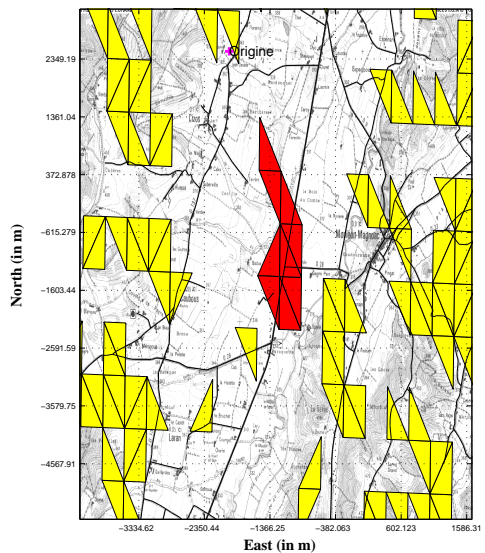

Fig. 4. Terrain masks at the current time $t_{k}=1$.

of the undetected areas, we have added a fixed terrain mask (the red triangles in the figure 4) in order to have the targets 1 to 5 in the same terrain mask.

\section{Filter Parameters}

In order to evaluate the algorithm performances we compare the VS IMM SB-MHT with an usual IMM SB-MHT presented in [15]. The parameters of the IMM are the following: three motion models are considered, one constant velocity motion model with a small standard deviation (fixed at $0.05 \mathrm{~m} \cdot \mathrm{s}^{-2}$ ), another constant motion model with a high standard deviation (fixed at $0.8 \mathrm{~m} \cdot \mathrm{s}^{-2}$ ) in order to palliate the target manoeuvre and a stop motion model (where the standard deviation is equal to zero). The VS IMMC is composed with three generics motion models : one constraint constant velocity model (the standard deviation along and orthogonal to the road segment are equal to $0.05 \mathrm{~m} \cdot \mathrm{s}^{-2}$ ), another constraint constant velocity model with a high standard deviation to adapt the dynamic to the target manoeuvre (the standard deviation along and orthogonal to the road segment are respectively equal to 0.8 $m \cdot s^{-2}$ and $0.4 m \cdot s^{-2}$ ) and a stop motion model. Those generics constraints motion models must be adapted following the road network topology. The SB-MHT parameters are taken in [15].

\section{Results}

The target tracking goal is to track for a long time the target with olny one track. Then, in order to evaluate the tack maintenance we use the track length ratio criterion for a target $n$ between the truth target trajectory length $l_{n}$ and the associated track length $L_{n}$ obtained in a run. According to 100 Monte-Carlo runs, we obtain for each track the average track length ratio $(\forall n \in\{1, \ldots, 6\})$ :

$$
R_{n}=\sum_{k=1}^{100} \frac{l_{n}}{100 \cdot L_{n}}
$$

In addition for each algorithm (VS IMMC or IMM) we have two length ratios. The ratio length noted $\mathbf{1}$ is the track length ratio obtained without ambiguity association (i.e. the

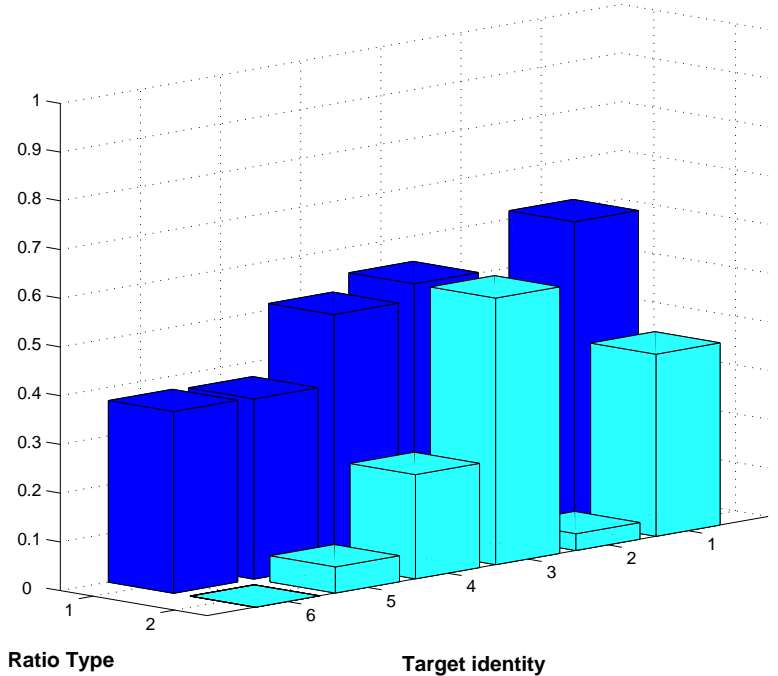

Fig. 5. IMM ratio track length for the 6 targets and for the 2 types of ratio.

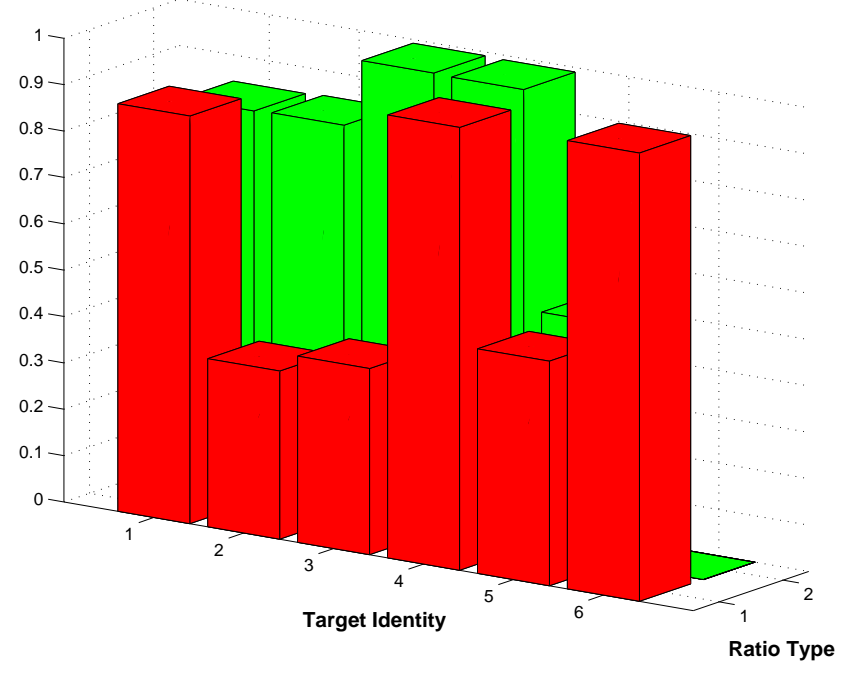

Fig. 6. VS IMMC ratio track length for the 6 targets and for the 2 types of ratio.

track is obtained after the decision described in the subsection IV-B). The second ratio length noted $\mathbf{2}$ is the track length ratio obtained with ambiguity association (i.e. the track has been generated by several sentinels and no decision can be taken on the track continuity).

On the figure 5, we can see that for each targets the associated track ratio is never equal to one. That implies the systematic tracks lost with the IMM. In fact when the tracks of the targets 1 to 5 enter in the terrain mask the tracks becomes in a sentinel configuration and the sentinels are placed at the exit of the mask. But the tracks are not constrained on the road and evolve in the last updated direction. Then the targets follow the road network and the unconstraint sentinels are inevitably not placed at the road proximity. When the targets 
appear through their MTI reports, the sentinels are far away in order to be attached to the new tracks generated by the MTI reports. That is why the track type length ratio 2 is low: there are few new tracks in which the sentinel information exists. On the other hand, the track length ratio 2 of the VS IMMC (see figure 6) is near to one for the target 1 to 4. Given that the tracks are constrained to the road network the sentinels are placed at the proximity of the target MTI report. So the new tracks contain the information that this track could be the continuation of the old track associated to the sentinel. In addition, the track length ratio type 1 of the tracks 1 and 4 is high that means that the algorithm has taken the decision to declare the new track like being the old track continuation without ambiguity. However, for the targets 2 and 3 no decision can be taken because in the corresponding new track there is an ambiguity, in the manner that each new track can be attached to several sentinels (either the sentinel associated to the target 2 or the sentinel associated to the target 3 ). For the target 5 , we can see that the track length ratio type 1 and 2 is low. This is the weakness of our approach. The target 5 enters in the terrain mask just when the target 1 leaves the mask. A new track is created with the target 1 MTI reports and the sentinel is attached to this track. Sometimes in a run, the target 5 track is also associated to this MTI report. After few scans the SB-MHT decides that the track associated to the sentinel is more probable than the track associated previously to the target 5 and this last one is lost. That explains this bad result. The figure 7 represents the Root Mean Square Error of the targets 1 and 4 obtained with the IMM SB-MHT and the VS IMMC SB-MHT. We can see that the RMSE is better with the VS IMMC than the IMM. However when the targets move in the terrain mask between $150 s$ and $230 s$, the RMSE of the VS IMMC increases. This is due to the last updated state residual error which is propagated in the mask. Nevertheless, the predicted error is compensated by the sentinel activation because the sentinel is waiting for a new MTI report. Another weakness of our approach is the target manoeuvre in the terrain masks. If the target manoeuvres quickly, the motion models are not adapted because in the terrain mask, each predicted state is propagated and not updated. The manoeuvre can be not detected. That is why the tracks could be lost is the associated targets manoeuvre quickly in the terrain masks.

\section{CONCLUSION}

In this paper, we have presented a method to track multiple ground targets with terrain obscuration. We assume that the non-detection of target is either due to stop of the target or the radial velocity under the MDV or the terrain mask. We have defined a perceivability probability to detect the obscuration areas. However, the usuals algorithms are not adapted to the track maintenance if several targets enter and exit in same terrain mask. So, a "sentinel" solution has been developed to modelize the uncertainty on the track continuation if several targets leave the same terrain mask. This approach improves the track maintenance expected if the targets manoeuvre quickly in the terrain mask. In this case it is difficult to
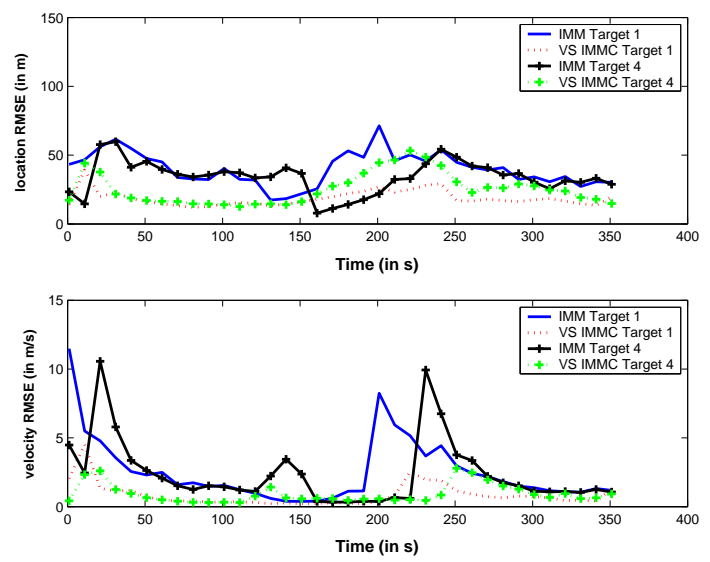

Fig. 7. Root Mean Square Error of the targets 1 and 4

discriminate the targets. That is why, in a future work, we must reduce the terrain mask areas by using several GMTI sensors and use the fusion with heterogeneous information in order to obtain the "type" information on a track.

\section{REFERENCES}

[1] B. Pannetier, K. Benameur, V. Nimier and M. Rombaut, "VS-IMM using road map information for a ground target tracking", Proceedings of Information Fusion 2005, vol. 1, Jul. 2005.

[2] B. Pannetier, K. Benameur and M. Rombaut, "Multiple ground target tracking with a GMTI sensor", Proceedings IEEE of multisensor fusion and integration for intelligent Systems 2006, pp. 230-236, Sept. 2006.

[3] T. Kirubarajan and Y. Bar-Shalom, "Tracking evasive move-stop-move targets with an MTI radar using a VS-IMM estimator", IEEE Transactions on aerospace and electronic systems, vol. 39, no. 3, pp. 1098-1103, Jul. 2003.

[4] T. Connare and E. Blasch, "Improving track maintenance through group tracking", Proceedings of the worshop on estimation, tracking and Fusion, A tribute to Yakoov Bar-Shalom, Monterey, CA. 2001.

[5] L. Lin, Y. Bar-Shalom and T. Kirubarajan, "New assignment based data association for tracking move-stop-move targets", IEEE Transactions on aerospace and electronic systems, vol. 409, no. 32, pp. 714-725, Apr. 2004.

[6] J. Dezert, L. Ning, X. Rong Li, "A new formulaion of IPDAF for tracking n clutter", Proceedings of European Control Conference, Sep. 1999.

[7] E. Blasch and C. Yang, "Ten methods to fuse GMTI and HRRR measurements for joint tracking aand identification", Proceedings of Information Fusion 2004, pp. 1006-1013, Jul. 2004.

[8] T. Kirubarajan, Y. Bar-Shalom, K.R. Pattipati and I. Kadar, "Ground target tracking with topography-basd variable structure IMM estimator", Proceedings of SPIE, signal and data processing of small targets, vol. 3373, pp. 222-233, Jul. 1998.

[9] J. G. Wang, T. Long and P.K. He, "A New Method of Incorporating Radial Velocity Measurement into Kalman Filter" ,Proccedings of signal Processing, no. 18, pp. 414-416, 2002.

[10] D.F. Bizup and D.E. Brown, "The over extended Kalman filter - Don't use it !", Proceedings of Information Fusion 2003, Jul. 2003.

[11] B. Pannetier, K. Benameur, V. Nimier and M. Rombaut, "Ground target tracking with road constraint", Proceedings of SPIE, sensor fusion and target recognition XIII, volume 5429, Apr. 2004.

[12] Y. Bar Shalom and D. Blair, Multi-Target Multi-Sensor tracking: Applications and Advances , Artech House, Vol. III, 2000.

[13] D. Simon and T.L. Chia, "Kalman filtering with state equality constraints", IEEE Transactions on aerospace and electronic systems, vol. 38, no. 1, pp. 128-136, Jan. 2002.

[14] Y. Bar Shalom, X.R. Li and T. Kirubarajan, Estimation with applications to tracking navigation: algorithms and software for informatio extraction , New York, Wiley, 2001.

[15] S.S. Blackman R. Popoli, Design and analysis of modern tracking systems , Artech House, 1999. 